\title{
Notoginseng root enhances healing in imiquimod-induced psoriasis mice model via anti-inflammatory and anti- proliferative properties
}

\author{
Yangqi Li ${ }^{1}$, Xianglong Dai ${ }^{1}$, Jinliang $\mathrm{Li}^{2}$, Jinghang Deng ${ }^{1}$, Changlan $\mathrm{Wan}^{3}$, Xia \\ $\mathrm{Xu}^{1}$, Fengxing Liang ${ }^{2}$, Fei Wang ${ }^{1}$, Jinbao Zhong ${ }^{2 \star}$ \\ ${ }^{1}$ Department of Dermatology, ${ }^{2}$ Department of Traditional Chinese Medical Dermatology, ${ }^{3}$ Department of Dermatology, \\ Guangzhou Institute of Dermatology, Guangzhou, Guangdong 510095, China
}

*For correspondence: Email: jinbaozhong68@hotmail.com; Tel: 0086-020-83583564

Sent for review: 13 March $2018 \quad$ Revised accepted: 19 November 2018

\begin{abstract}
Purpose: To evaluate the beneficial effect of Panax notoginseng (PN) gel against imiquimod-induced psoriasis in a mice model.

Methods: Psoriasis was induced by topical application of imiquimod cream (5\%) on the shaved skin of mice for 7 days. PN group received PN gel (1\%) twice a day with imiquimod cream (5\%) once a day for one week. The effect of PN gel was estimated by scoring skin thickness, scaling and erythema. Reverse transcription polymerase chain reaction (RT-PCR) was used for the determination of the expressions of inflammatory mediators in skin tissues of mice. Moreover, the severity of inflammation was determined by histopathological and immunohistochemical assessment of skin tissues.

Results: The severity of inflammation and the expressions of inflammatory mediators were significantly reduced in $P N$ gel-treated group, relative to the negative control group. Treatment with $P N$ gel attenuated the histopathology of skin tissue in the imiquimod-induced psoriatic mice, and significantly decreased the level of intercellular adhesion molecule (ICAM-1), when compared to the negative control group.

Conclusion: These results show that PN gel attenuates psoriasis in imiquimod-induced psoriasis mice model by decreasing skin inflammation. Thus, $P N$ gel may be suitable for the management of psoriasis.
\end{abstract}

Keywords: Psoriasis, Panax notoginseng, Inflammatory mediators, Imiquimod, Intercellular Adhesion Molecule-1

\begin{abstract}
This is an Open Access article that uses a funding model which does not charge readers or their institutions for access and distributed under the terms of the Creative Commons Attribution License (http://creativecommons.org/licenses/by/4.0) and the Budapest Open Access Initiative (http://www.budapestopenaccessinitiative.org/read), which permit unrestricted use, distribution, and reproduction in any medium, provided the original work is properly credited.

Tropical Journal of Pharmaceutical Research is indexed by Science Citation Index (SciSearch), Scopus, International Pharmaceutical Abstract, Chemical Abstracts, Embase, Index Copernicus, EBSCO, African Index Medicus, JournalSeek, Journal Citation Reports/Science Edition, Directory of Open Access Journals (DOAJ), African Journal Online, Bioline International, Open-J-Gate and Pharmacy Abstracts
\end{abstract}

\section{INTRODUCTION}

Psoriasis is a chronic inflammatory skin disease which affects about $2 \%$ of the world's population [1]. It is characterized by leukocyte infiltration, dilatation of capillaries and hyper-proliferation of keratocytes [2]. The pathogenesis of psoriasis is thought to be due to environmental and genetic factors [3]. Studies have revealed that inflammatory pathways play an important role in the pathogenesis of psoriasis due to their involvement in the activation of the proliferation of keratocytes and infiltration of immune cells [4]. In psoriasis, the production of chemokines and 
cytokines activates the NF-KB signaling pathway [5]. Psoriatic patients suffer social and emotional disabilities due to the visibility of the psoriatic lesions [6]. There are several therapies currently available for the management of psoriasis. These are phototherapy, and systemic and topical agents. However, none of these therapies can cure this skin disorder completely. In addition, each therapy has its own limitations. Thus, it is important to develop new therapeutic approaches for the management of psoriasis.

Traditional Chinese medicine has shown promising potential in the management of psoriasis. Panax notoginseng belongs to the family Araliaceae. In China, it is traditionally used for the management of hemorrhage [7]. Some compounds isolated from Panax notoginseng i.e. notoginsenoside and ginsenosides possess pharmacological activities such as anti-myocardial ischemia, anti-cancer, immunomodulatory, hepatoprotective and antifibrotic properties [8 - 10]. Panax notoginseng has been reported to prevent brain injury by attenuating altered levels of oxidative stress and inflammatory mediators [11].The present study evaluates the healing effect of PN against psoriasis, and the mechanisms involved.

\section{EXPERIMENTAL}

\section{Animals}

Female BALB/c mice, aged 6 - 8 weeks, and weighing 20 - $25 \mathrm{~g}$, were procured from Shanghai Medical College, China. All animals were kept under standard conditions as per guidelines. They were acclimatized to laboratory conditions for 7 days, and allowed free access to normal standard chow diet and tap water. The experiments used in this investigation were approved by the institutional ethical committee of Guangzhou Institute of Dermatology, China (approval no. IAEC/GID/2016/21). The study followed the guidelines of the Association for the Assessment and Accreditation of Laboratory Animal Care International (AAALAC) for experimentation and animal use [12].

\section{Extraction of Panax notoginseng}

The roots of Panax notoginseng were procured and authenticated by a taxonomist at Guangzhou Institute of Science, China. The roots were dried under the shade and coarsely powdered. The dried powder was extracted by maceration using 80:20 volume ratio of methanol: water. The solvent mixture was evaporated in vacuo to produce a dry extract. The percentage yield of the extract was $20.7 \%$.

\section{Preparation of Panax notoginseng gel}

The preparation of $1 \%$ Panax notoginseng gel formulation was done as per the procedure reported in a previous study [13]. The gel was prepared by mixing hydroxypropylcellulose (HPC) in distilled water at ambient temperature. Then, the dried Panax notoginseng extract was dissolved in $95 \%$ of ethanol and the resultant solution was mixed with the HPC solution. The mixing was carried on continuously until the formation of gel.

\section{Induction of psoriasis}

Psoriasis was induced by topical application of imiquimod cream (5\%) on the shaved skin of mice for 7 days. The rats were assigned to three groups: negative control group which received imiquimod cream (5\%) topically on the shaved epidermal area for 7 days, PN group which received PN gel (1\%) twice daily, along with imiquimod cream (5\%) once a day for 7 days, and control group. The severity of inflammation was estimated by determining psoriasis area and severity index (PASI) as per previously reported method [13]. Thickness, scaling, erythema and cumulative score of skin-like parameters were determined, and the degree of inflammation (from a scale of 0 - 4) was estimated separately for each parameter.

\section{Real-time PCR}

The mice were sacrificed by decapitation, and TRIzol reagent was used to extract the mRNA from the isolated skin. The reverse transcription of total RNA $(2 \mu \mathrm{g})$ to cDNA was done with iScript cDNA Synthesis kit, while SYBR Green Master Mix was used for quantitative RT-PCR as per the manufacturer's instructions. Primer specificity of the amplification was evaluated by using melting curve analysis. The expressions of IL-23, ICAM-1, TNF- $\alpha$ and IL-8 were estimated, with GAPDH as an internal control.

\section{Immunohistochemical studies}

Following fixation in $4 \%$ paraformaldehyde, paraffin was added to the isolated skin samples from each animal. The skin samples were sectioned into $4 \mu \mathrm{m}$ thickness and stained with hematoxylin eosin ( $\mathrm{H} \& \mathrm{E})$. The tissue sections were observed for histopathological changes under a light microscope. Moreover, histochemistry was carried out by incubating the tissue sections with anti-mouse ICAM- 1 antibody (1: 200 dilution) and later with biotin-conjugated goat anti-rabbit IgG for $50 \mathrm{~min}$. Thereafter, the tissue sections were subjected to DAB and 
hematoxylin staining, and observed under the light microscope.

\section{Statistical analysis}

Data are expressed as mean $\pm \operatorname{SD}(n=6)$. Statistical analysis was performed using one way ANOVA. Post-hoc comparison of means was carried out by Dunnett's post hoc test (Gradpad prism 6.1., CA, USA). The level of statistical significance was set at $p<0.05$.

\section{RESULTS}

\section{Effect of Panax notoginseng gel on severity of inflammation}

The effect of Panax notoginseng gel on severity of inflammation in imiquimod-induced psoriasis mouse model is shown in Figure 1. In the exposed area of the control group, the skin was smooth, with vessels visible underneath. There were significant increases in the thickness of skin, scaling and erythemia on the shaved skin of
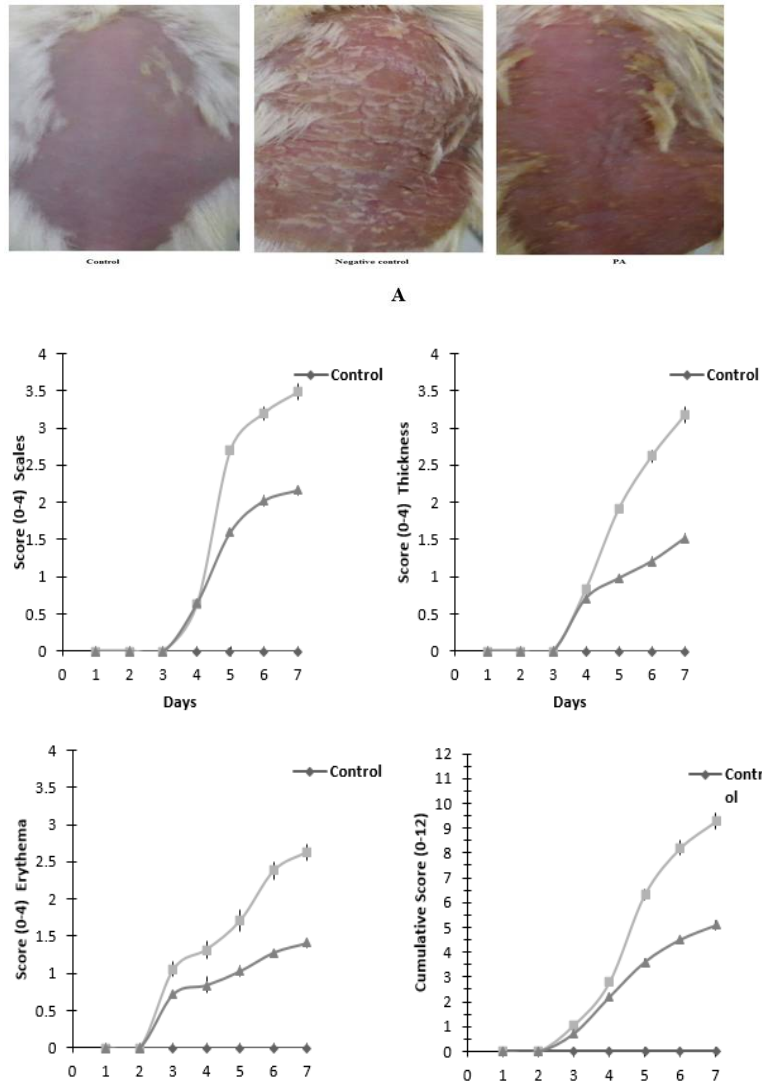

Days

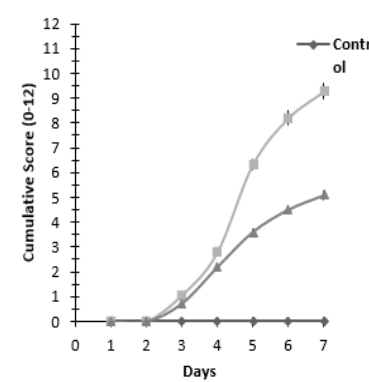

Figure 1: Effect of Panax notoginseng gel on severity of inflammation in imiquimod-induced psoriasis mouse model. Data are presented as mean $\pm \operatorname{SD}(n=6),{ }^{\star *} p<$ 0.01 , compared to the control group; ${ }^{\#} p<0.01$, compared to the negative control group negative control group, when compared to the control group. In addition, the exposed area showed erythema and thickened layers of skin. However, treatment with PN attenuated the skin lesions on the exposed area, and also attenuated the level of skin thickness, scaling and erythema in the imiquimod-induced psoriasis rat model (Figure 1B).

\section{Effect of Panax notoginseng gel on expressions of inflammatory mediators}

The effect of Panax notoginseng gel on the expressions of inflammatory cytokines in the skin tissue of imiquimod-induced psoriasis mouse model is shown in Figure 2. There were significant increases in the expressions of IL-8, TNF- $\alpha$, IL-17 and ICAM- 1 in the skin tissues of the negative control group, relative to the control group $(p<0.05, p<0.01)$. However, application of PN gel on the exposed skin of imiquimodpsoriatic mice significantly reduced the expressions of IL-8, TNF- $\alpha$, IL-17 and ICAM-1, when compared to the negative control group.

\section{Effect of Panax notoginseng gel on skin inflammation}

The effect of Panax notoginseng gel on the level of skin inflammation was estimated by histopathological and immunohistochemical analyses. There was a higher degree of epidermal layer thickening in the negative control group than in the control group (Figure 3). Histopathological changes in the skin tissue are shown in (Figure 3A). Acanthosis was found in the skin, and granular and horny layer were absent in the skin tissues of the negative control group. However, treatment with PN attenuated the lesions and skin inflammation brought about by imiquimod-induced psoriasis.

Immunohistochemical evaluation revealed that the expression of ICAM-1 protein was significantly downregulated in the skin tissue of the PN-treated group, when compared to the negative control group (Figure 3B). Epidermal thickness was significantly enhanced in the negative control group, relative to the control group. However, treatment with PN gel significantly attenuated the enhanced thickness of epidermal layer in imiquimod-induced psoriasis mice (Figure 3C).

\section{DISCUSSION}

Psoriasis is an inflammatory skin disorder. Studies have revealed the promising potential of alternative medicine in the management of inflammatory skin disorders [14]. The present 

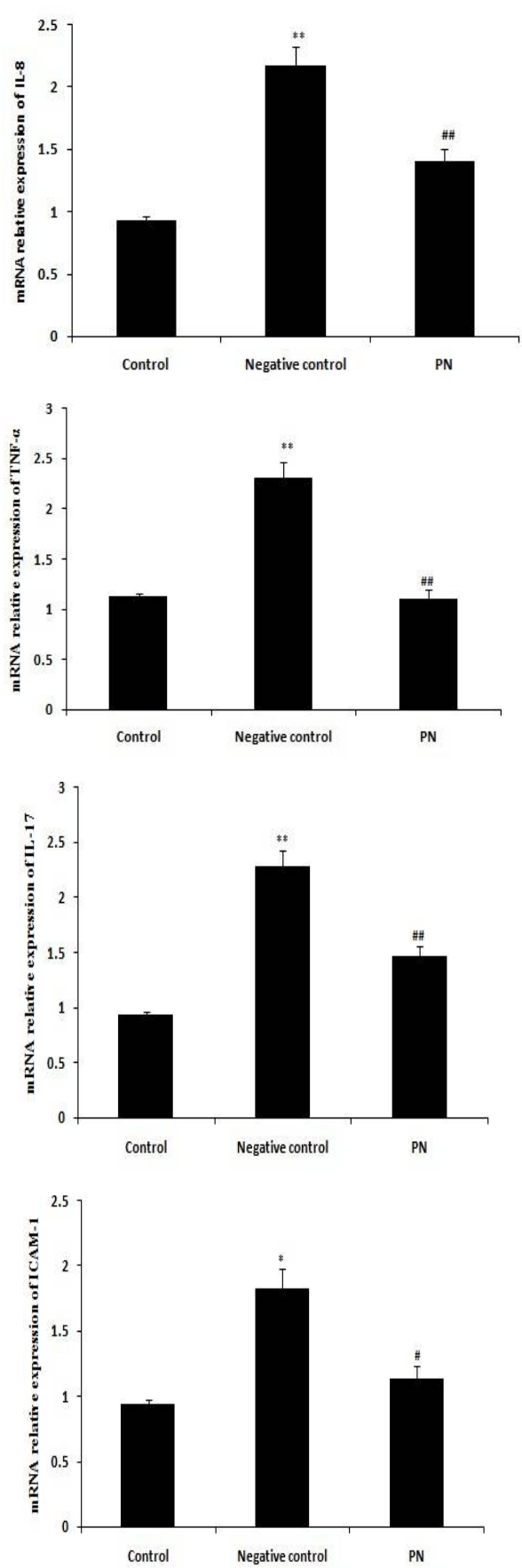

Figure 2: Effect of Panax notoginseng gel on the expressions of inflammatory cytokines in the skin tissue of imiquimod-induced psoriasis mouse model. Data are presented as mean \pm SD $(n=6),{ }^{* *} p<0.01$, compared to the control group, ${ }^{\# \#} p<0.01$, relative to the negative control group

study evaluated the beneficial effect of Panax notoginseng gel against psoriasis in rats. Psoriasis was induced by topical application of imiquimod on the shaved area of mouse skin, and PASI was estimated by scoring skin thickness, scaling and erythema. Moreover, RTPCR was used for the determination of expressions of inflammatory mediators in the mice skin tissues.
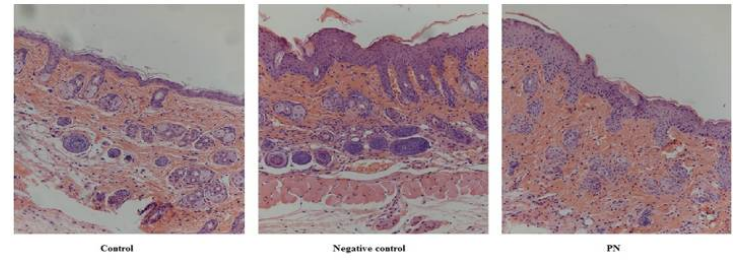

$\mathbf{A}$
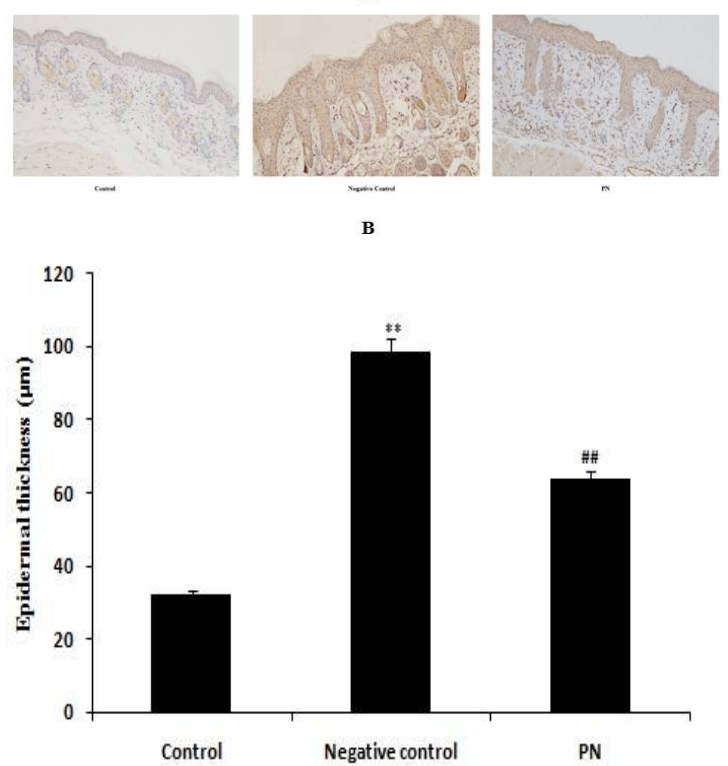

C

Figure 3: Effect of Panax notoginseng gel on the level of skin inflammation in imiquimod-induced psoriasis mouse model. A: Histopathology of skin $(H \& E)$; $B$ : Immunohistochemical analysis of ICAM-1; C: Epidermal thickness. Results are presented as mean \pm SD $(n=6) .{ }^{* *} p<0.01$, compared to the control group; $\# p<0.01$, compared to the negative control group

It has been reported that topical application of imiquimod produces skin inflammation which resembles that of psoriasis [15]. In this study, it was also revealed that inflammation and expressions of inflammatory mediators were significantly enhanced in the negative control group, when compared with the control group. However, treatment with PN gel attenuated the imiquimod-induced inflammation. Inflammatory mediators such as ICAM-1, IL-17, IL-8 and TNF$\alpha$ are involved in the pathogenesis of psoriasis [16]. Studies have demonstrated that IL-17 is involved in psoriatic lesions, while TNF- $\alpha$ activates NF-KB in psoriatic patients [17]. In the present study, treatment with PN gel significantly attenuated the altered expressions of inflammatory mediators in the skin tissue of imiquimod-induced psoriatic mice. This finding is consistent with previously published data [18].

Histopathologic studies also revealed that application of PN gel attenuated the imiquimodinduced skin lesions and epidermal layer thickening in the psoriatic mice. Again, this result is in agreement with previous reports [19]. 
Immunohistochemical analysis showed that the level of ICAM-1 was significantly reduced in the PN gel-treated group, when compared to the negative control group.

\section{CONCLUSION}

The findings of this study demonstrate that PN gel treatment attenuates imiquimod-induced psoriasis in mice by decreasing skin inflammation. Thus, PN has a potential for application in the management of psoriasis.

\section{DECLARATIONS}

\section{Acknowledgement}

All the authors of this manuscript are thankful to Building a Strong Provincial Scientific Research in Guangdong (no. 20142119 1/10), Guangzhou Traditional Chinese Medicine and Traditional Chinese and Western Medicine Science and Technology Project (no. 20142A011020 1/10), and Guangzhou Traditional Chinese Medical Health Science And Technology Project (no. 20132A0110231/10) for providing funds for this work.

\section{Conflict of Interest}

No conflict of interest associated with this work.

\section{Contribution of Authors}

The authors declare that this work was done by the authors named in this article and all liabilities pertaining to claims relating to the content of this article will be borne by them.

\section{REFERENCES}

1. Griffiths CEM, van de Kerkhof $P$, Czarnecka-Operacz M. Psoriasis and Atopic Dermatitis. Dermatol Ther (Heidelb) 2017; 7(Suppl 1): 31-41.

2. Gu X, Reagan AM, McClellan ME, Elliott MH. Caveolins and caveolae in ocular physiology and pathophysiology. Prog Retin Eye Res 2017; 56: 84- 106.

3. AlShobaili HA, Shahzad M, Al-Marshood A, Khalil A, Settin A, Barrimah I. Genetic Background of Psoriasis. Int J Health Sci (Qassim) 2010; 4 (1): 23-29.

4. Smith MM, Melrose J. Proteoglycans in Normal and Healing Skin. Adv Wound Care (New Rochelle) 2015; 4(3): 152-173.

5. Li R, Wang J, Wang X, Zhou J, Wang M, Ma H, Xiao S, Increased $\beta T r C P$ are associated with imiquimodinduced psoriasis-like skin inflammation in mice via NFкB signaling pathway. Gene 2016; 592(1): 164-171.
6. Ferreira BIRC, Abreu JLPDC, Reis JPGD, Figueiredo AMDC. Psoriasis and Associated Psychiatric Disorders: A Systematic Review on Etiopathogenesis and Clinical Correlation. J Clin Aesthet Dermatol 2016; 9(6): 36-43.

7. Duan L, Xiong $X$, Hu J, Liu Y, Li J, Wang J. Panax notoginseng Saponins for Treating Coronary Artery Disease: A Functional and Mechanistic Overview. Front Pharmacol 2017; 8: 702.

8. Geng J, Peng W, Huang Y, Fan H, Li S., GinsenosideRg1 from Panax notoginseng prevents hepatic fibrosis induced by thioacetamide in rats. Eur $J$ Pharmacol 2010; 634(1-3): 162-69.

9. Wang $C-Z$, Xie JT, Fishbein $A$, Aung $H H$, He $H$, Mehendale SR, He TC, Du W, Yuan CS. Antiproliferative Effects of Different Plant Parts of Panax notoginseng on SW480 Human Colorectal Cancer Cells. Phytother Res 2009; 23(1): 6-13.

10. Wang CZ, Xie JT, Zhang B, Ni M, Fishbein A, Aung HH, Mehendale SR, Du W, He TC, Yuan CS., Chemopreventive effects of Panax notoginseng and its major constituents on SW480 human colorectal cancer cells. Int J Oncol 2007; 31(5): 1149-56.

11. Huang JL, Jing $X$, Tian $X$, Qin MC, $X u Z H, W u D P$, and Zhong ZG, Neuroprotective Properties of Panax notoginseng Saponins via Preventing Oxidative Stress Injury in SAMP8 Mice. Evid Based Complement Alternat Med. 2017 (2017), Article ID 8713561.

12. Guide for the Care and Use of Laboratory Animals: Eighth Edition Committee for the Update of the Guide for the Care and Use of Laboratory Animals; National Research Council. 2010; ISBN: 0-309-15401-4

13. Sun J, Zhao Y, Hu J. Curcumin Inhibits ImiquimodInduced Psoriasis-Like Inflammation by Inhibiting IL1 beta and IL-6 Production in Mice. Tanowitz HB, ed. PLOS ONE. 2013; 8(6): e67078.

14. Tabassum N, Hamdani M. Plants used to treat skin diseases. Pharmacogn Rev 2014; 8(15): 52-60.

15. Mori H, Arita K, Yamaguchi T, Hirai M, Kurebayashi $Y$. Effects of Topical Application of Betamethasone on Imiquimod-induced Psoriasis-like Skin Inflammation in Mice. Kobe J Med Sci 2016; 62(4): E79-E88.

16. Bai F, Zheng, W., Dong, Y., Wang, J., Garstka, M. A., Li, R., An, J., Ma, H. Serum levels of adipokines and cytokines in psoriasis patients: a systematic review and meta-analysis. Oncotarget 2018; 9(1): 1266-1278.

17. Nograles KE, Davidovici B, Krueger JG. New insights in the Immunologic Basis of Psoriasis. Semin Cutan Med Surg 2010; 29(1): 3-9.

18. Oka T, Sugaya M, Takahashi N, Takahashi T, Shibata S, Miyagaki T, Asano Y, Sato S., CXCL17 Attenuates Imiquimod-Induced Psoriasis-like Skin Inflammation by Recruiting Myeloid-Derived Suppressor Cells and Regulatory T Cells. J Immunol. 2017; 198(10): 38973908.

19. Dou R, Liu Z, Yuan X, Xiangfei D, Bai R, Bi Z, Yang $P$, Yang $Y$, Dong $Y$, Su W, Li D, Mao C. PAMs ameliorates the imiquimod-induced psoriasis-like skin disease in mice by inhibition of translocation of NF-KB and Trop J Pharm Res, December 2018; 17(12): 2369 
